Short Report

\title{
Serum albumin is an early predictor of complications after liver surgery
}

\author{
Ismail Labgaa, Gaëtan-Romain Joliat, Nicolas Demartines*, Martin Hübner \\ Department of Visceral Surgery, University Hospital of Lausanne (CHUV), Switzerland
}

\section{A R T I C L E I N F O}

\section{Article history:}

Received 4 October 2015

Accepted 5 January 2016

Available online 9 January 2016

\section{Keywords:}

Biomarker

Liver surgery

Postoperative complications

Serum albumin

\begin{abstract}
A B S T R A C T
Background: The morbidity associated with liver surgery remained substantially high despite considerable surgical and anesthetic improvements. The unmet need of accurate biomarkers to predict postoperative complications is widely accepted.

Aims: This pilot study aimed to assess serum albumin as a surrogate marker of surgical stress and to test its potential predictive role for postoperative complications.

Methods: This retrospective pilot study included 106 patients who underwent liver surgery between 2010 and 2014. Serum albumin levels were measured pre- and post-operatively. Maximal albumin decrease (Alb $\Delta \min$ ) was correlated with complications.

Results: Serum albumin rapidly dropped after surgery. Alb $\Delta$ min was significantly increased in patients with complications $(14.5 \pm 6.0 \mathrm{~g} / \mathrm{L}$ vs. $10.3 \pm 7.2, p=0.009)$. On multivariate analysis, ASA III/IV $(p=0.016)$ and Alb $\Delta \min (p=0.037)$ were the only predictors of overall complications.

Conclusion: Early postoperative drop of serum albumin reflects the intensity of the surgical stress and may predict complications after liver surgery. Serum albumin is a biomarker displaying precious features and deserving further prospective investigations.
\end{abstract}

@ 2016 Editrice Gastroenterologica Italiana S.r.l. Published by Elsevier Ltd. All rights reserved.

\section{Introduction}

Over the last decades, surgical, anesthetics and perioperative improvements have permitted to safely offer liver resection to more high-risk patients [1]. Whereas a decreased postoperative mortality rate of less than $5 \%$ was observed [2], the postoperative morbidity including major complications remained high, reaching 50\% [2].

The intensity of the metabolic stress triggered by surgery presumably correlates with the risk of complications [3]. Of note, several measures were reported to successfully attenuate the overshooting surgical stress response and to reduce morbidity [4]. Surrogate markers for the early stress response would therefore be appealing tools to better tailor the management of patients undergoing surgery.

Recently, Mantziari et al. demonstrated that serum albumin showed a rapid drop after major abdominal surgery, reflecting the amplitude of the surgical stress and correlating with the risk of adverse outcomes [5].

\footnotetext{
* Corresponding author at: Department of Visceral Surgery, University Hospital CHUV, 1011 Lausanne, Switzerland. Tel.: +41 2131424 00; fax: +41213142411. E-mail address: demartines@chuv.ch (N. Demartines).
}

The aim of the present study was therefore to assess the potential role of serum albumin concentration as a surrogate marker of stress response and predictor of adverse outcomes after liver surgery.

\section{Methods}

This retrospective cohort study was conducted in the department of Visceral Surgery, University Hospital of Lausanne (CHUV). The study was conducted in accordance with the STROBE criteria (http://strobe-statement.org/) and registered under www. researchregistry.com (UIN: 542).

\subsection{Patients}

One hundred seventy-four patients who underwent elective liver surgery between June 2010 and July 2014 were identified from our prospective database. Only patients with available preand post-operative (POD 0 or POD 1) serum albumin concentrations were included. Age $<18$ years, immunosuppressive treatment and emergency surgery were considered as exclusion criteria. As a result, 106 patients were considered in this analysis. 


\subsection{Data collection}

Serum albumin $(\mathrm{g} / \mathrm{L})$ levels were measured in a fasting state, preand post-operatively according to standardized guidelines of the Hospital. Samples on POD 0 were collected 4-6 hours after surgery. Relevant demographics, surgical details and clinical outcomes were prospectively collected and anonymized in a computerized database. Operation duration was measured from incision to skin closure by the anesthetists. Intraoperative blood loss was estimated by measuring the volume of aspirated fluid and soaked gauzes, jointly by the anesthetists and the surgeons. The extent of the resection was assessed according to Couinaud classification: minor resection is $<3$ segments; major resection is $\geq 3$ segments. The Clavien Classification was used to grade postoperative complications.

\subsection{Statistics}

Categorical variables were compared using Chi-squared test or Fisher's exact test as appropriate. Continuous variables were compared using two-sample Student $t$-test. A $p$ value of $<0.05$ was considered significant; variables with significance $<0.1$ were included in multivariate analyses. Data analyses were performed using SPSS v20 statistical software (Chicago, IL).

\section{Results}

\subsection{Demographics, surgery and outcomes}

Median age of the cohort was 64 years, with $34.9 \%$ of women, and $67.9 \%$ of ASA I-II. Comorbidities like smoking, diabetes and cirrhosis accounted for $24.5 \%, 19.8 \%$ and $11.3 \%$, respectively. Major resections and open surgery represented $56.7 \%$ and $90.4 \%$, respectively. The overall mortality and morbidity were $1.9 \%$ and $67.9 \%$, respectively. Minor complications were reported in 67 (63.2\%) patients, while major ones arose in $21(19.8 \%)$ cases.

\subsection{Serum albumin response to surgical stress}

Fig. 1 illustrates the perioperative profile of serum albumin in patients with complication vs. those without complication. In both groups, the surgical stress induced a rapid response characterized by a significant drop of serum albumin on POD 0 , followed by a slow recovery phase on POD 1 . The amplitude of the prompt decrease of serum albumin was labeled Alb $\Delta$ min and calculated as the difference between the preoperative and the POD 0 concentrations of serum albumin. Postoperative albumin drop (Alb $\Delta$ min) appeared to be significantly amplified in patients

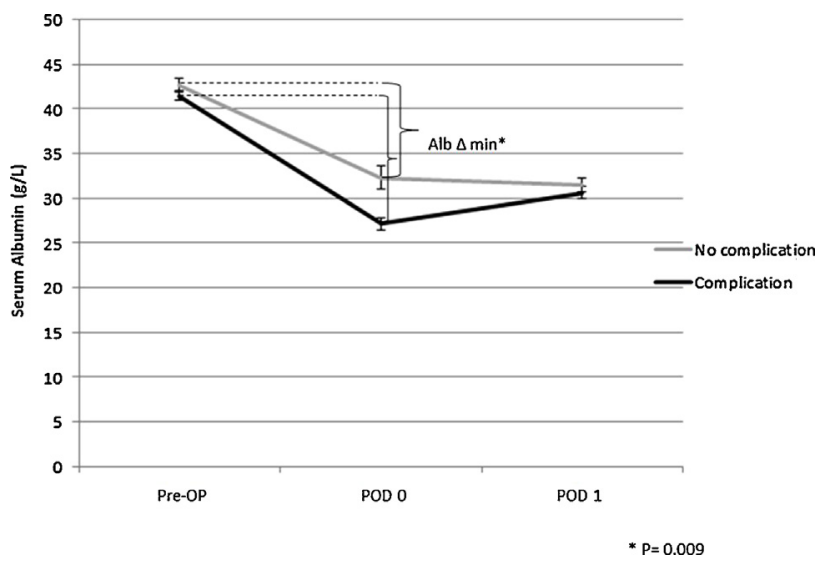

Fig. 1. Perioperative profile of serum albumin. Perioperative concentrations of serum albumin $(\mathrm{g} / \mathrm{L})$ in patients undergoing liver surgery, with complication (black) vs. with no complication (gray). Alb $\Delta$ min illustrated the postoperative drop of serum albumin and was calculated as the difference between the pre-operative and the POD 0 (4-6 hours after surgery) levels of serum albumin.

with complication, compared to patients without complication $(14.5 \pm 6.0$ vs. $10.3 \pm 7.2 \mathrm{~g} / \mathrm{L}, p=0.009)$.

\subsection{Serum albumin is a predictor of overall complications}

We further explored the influence of each variable on the occurrence of postoperative complications (Table 1). On univariate analysis, 4 factors were significantly associated with an increased risk of complications: ASA III/IV (HR=3.69, $p=0.016)$, BMI (HR1.13, $p=0.02$ ), blood loss (HR1, $p=0.041$ ) and Alb $\Delta \min$ $(\mathrm{HR}=1.11, p=0.01)$. The multivariate analysis only confirmed the significant impact of ASA III/IV $(\mathrm{HR}=7.46, p=0.016)$ and Alb $\Delta \min (H R=1.10, p=0.037)$. On further multivariate analysis (Table 2), Alb $\Delta$ min correlated with minor complications $(p=0.035)$, whereas no correlation was highlighted with major complications $(p=0.892)$.

\section{Discussion}

Serum albumin level rapidly dropped after liver surgery. The present results further suggested that the amplitude of this decrease (Alb $\Delta \min$ ) recapitulates the intensity of the surgical stress and is able to predict postoperative complications.

Although the adverse impact of preoperative hypoalbuminemia has been highlighted in liver surgery [1], data on early postoperative albumin are scarce. The potential interest of monitoring serum

Table 1

Predictors of overall postoperative complications after liver surgery.

\begin{tabular}{|c|c|c|c|c|c|c|}
\hline & \multicolumn{3}{|c|}{ Univariate } & \multicolumn{3}{|c|}{ Multivariate } \\
\hline & HR & $95 \% \mathrm{CI}$ & $p$ value & HR & $95 \% \mathrm{CI}$ & $p$ value \\
\hline Age & 1.00 & $0.96-1.04$ & 0.982 & & & \\
\hline Gender (men) & 1.33 & $0.57-3.11$ & 0.515 & & & \\
\hline ASA III/IV & 3.69 & $1.28-10.66$ & 0.016 & 7.46 & $1.46-38.1$ & 0.016 \\
\hline Cirrhosis & - & - & 0.999 & & & \\
\hline Cancer & 1.58 & $0.51-4.88$ & 0.426 & & & \\
\hline Smoker & 1.70 & $0.61-4.73$ & 0.31 & & & \\
\hline Diabetes & 2.20 & $0.68-7.15$ & 0.189 & & & \\
\hline BMI & 1.13 & $1.02-1.26$ & 0.02 & 1.13 & $0.96-1.32$ & 0.132 \\
\hline Surgery duration & 1.00 & $1.00-1.01$ & 0.057 & 1.00 & $0.99-1.01$ & 0.858 \\
\hline Blood loss & 1.00 & $1.00-1.002$ & 0.041 & 1.00 & 0.99-1.001 & 0.832 \\
\hline Major resection & 0.77 & $0.33-1.80$ & 0.541 & & & \\
\hline $\operatorname{Alb} \Delta \min$ & 1.11 & $1.03-1.21$ & 0.01 & 1.10 & $1.01-1.20$ & 0.037 \\
\hline
\end{tabular}


Table 2

The impact of Alb $\Delta$ min on complications.

\begin{tabular}{|c|c|c|c|c|c|c|}
\hline & \multicolumn{3}{|c|}{ Univariate } & \multicolumn{3}{|c|}{ Multivariate } \\
\hline & HR & $95 \% \mathrm{CI}$ & $p$ value & HR & $95 \% \mathrm{CI}$ & $p$ value \\
\hline \multicolumn{7}{|c|}{ Complications } \\
\hline Overall & 1.11 & $1.03-1.21$ & 0.01 & 1.1 & $1.01-1.20$ & 0.037 \\
\hline Minor (I-II) & 1.10 & $1.02-1.19$ & 0.013 & 1.11 & $1.01-1.22$ & 0.035 \\
\hline Major (III-V) & 1.00 & $0.92-1.08$ & 0.94 & 0.99 & $0.90-1.10$ & 0.892 \\
\hline
\end{tabular}

albumin in the perioperative phase of liver surgery is addressed for the first time, herein. Strikingly, serum albumin displays all the characteristics of an ideal biomarker: easy to use, can be repeated for monitoring, unexpansive, and it is modified in the early postoperative course. In addition, the role of serum albumin may not be limited to liver surgery, as supported by recent data on major abdominal surgery [5].

Main limitations of the present study are the small number of patients and its retrospective design. However, all eligible patients were included in the analysis. One may argue that the reported complications rate is higher than in comparable studies. Nevertheless, the lower mortality rate certainly favors thoroughly reported complications rather than questioning the perioperative management $[1,2]$.

In conclusion, serum albumin showed a rapid drop after liver surgery, which amplitude (Alb $\Delta \mathrm{min}$ ) is suggested to recapitulate the intensity of the surgical stress and may predict postoperative complications. This finding could be particularly relevant, since serum albumin can be measured early after surgery, when interventions to limit the overshooting stress could be initiated. A prospective study is currently underway (NCT02356484) in order to confirm or refute these preliminary findings.

\section{Conflict of interest}

None declared.

\section{Acknowledgments}

The authors would like to thank V. Addor and G. Jarrar for their contribution in the collection of data.

\section{References}

[1] Poon RT, Fan ST, Lo CM, et al. Improving perioperative outcome expands the role of hepatectomy in management of benign and malignant hepatobiliary diseases: analysis of 1222 consecutive patients from a prospective database. Annals of Surgery 2004:240:698-708, discussion 710.

[2] Breitenstein S, DeOliveira ML, Raptis DA, et al. Novel and simple preoperative score predicting complications after liver resection in noncirrhotic patients. Annals of Surgery 2010;252:726-34.

[3] Kohl BA, Deutschman CS. The inflammatory response to surgery and trauma. Current Opinion in Critical Care 2006;12:325-32.

[4] Hall R. Identification of inflammatory mediators and their modulation by strategies for the management of the systemic inflammatory response during cardiac surgery. Journal of Cardiothoracic and Vascular Anesthesia 2013;27:983-1033.

[5] Mantziari S, Hubner M, Coti-Bertrand P, et al. A novel approach to major surgery: tracking its pathophysiologic footprints. World Journal of Surgery 2015;39:2641-51. 